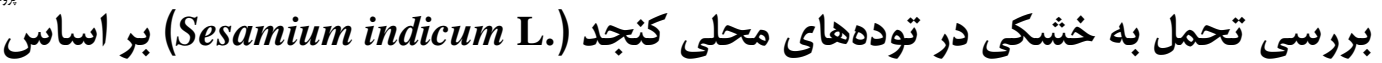

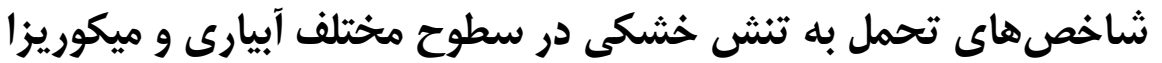

\author{
اسماعيل قلىنزاد' و رضا درويشزاده
}

ا - دانشيار، كروه علمى علوم كشاورزى، دانشكاه بيام نور، تهران، ايران (نويسنده مسوول: yahoo.com (gholinezhad1358)

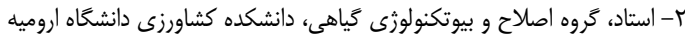

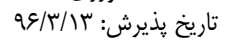
تاريخ دريافت:

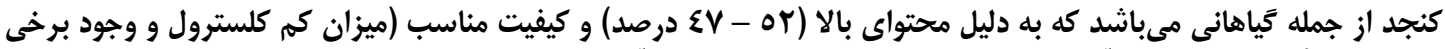

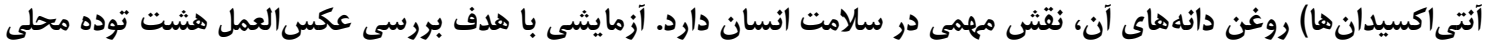

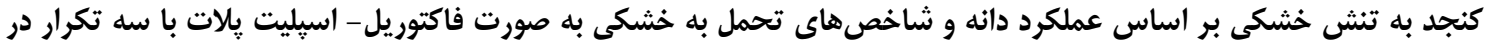

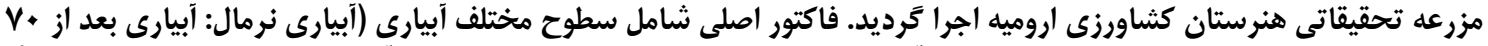

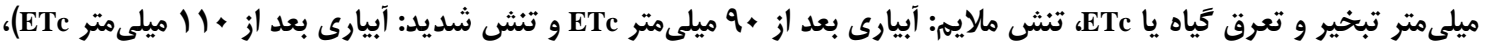

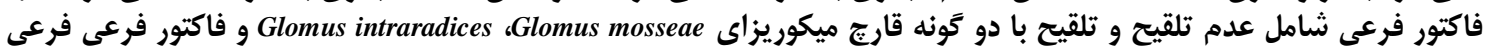

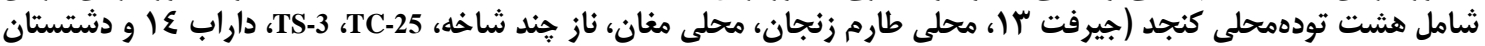

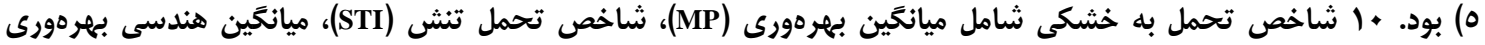

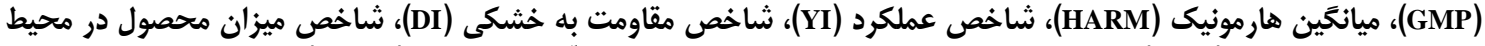

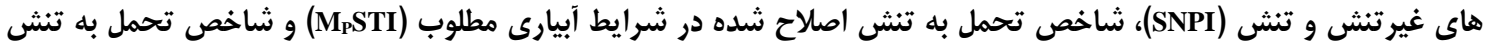

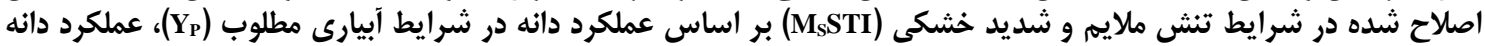

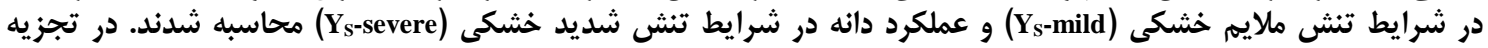

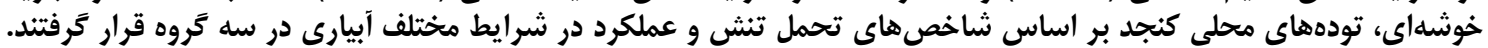

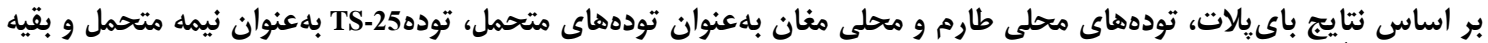

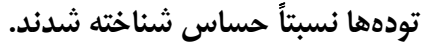

وازههاى كليدى: باىيلات، تجزيه خوشهاى، تنش خشكى، توده محلى، كنجد

كلوموس موسه سبب بهبود مقاومت بله تنش خشكى ومتى و جبران

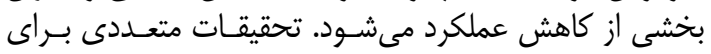

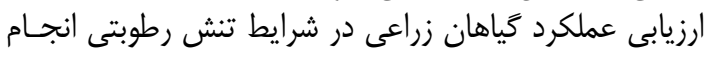

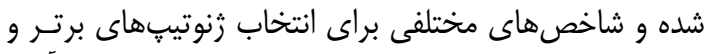

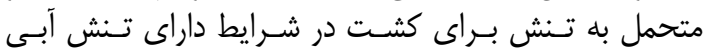

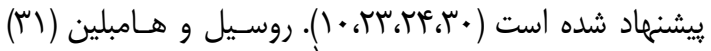

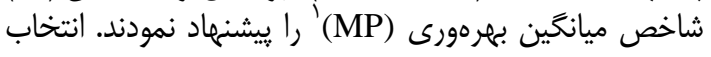

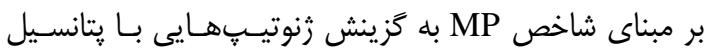

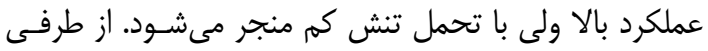

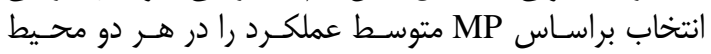

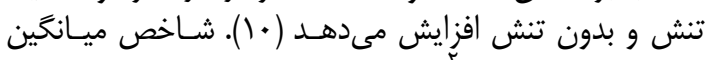

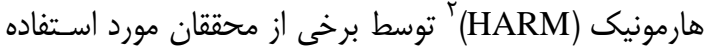

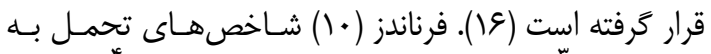

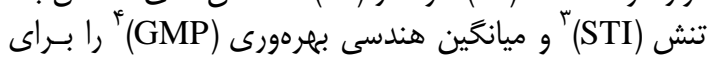

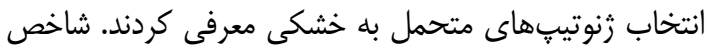

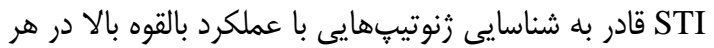

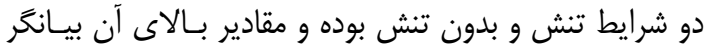

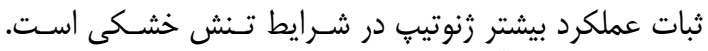

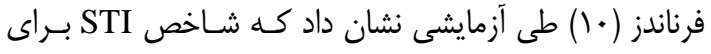

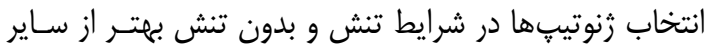

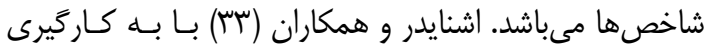

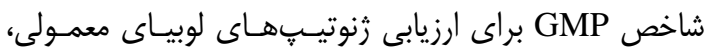

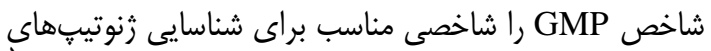

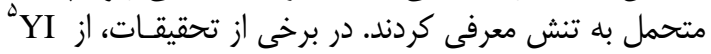

1-Mean Productivity

3- Stress Tolerance Index
كنجــد (Sesamun indicum L.) خيـاهى يكســاله و

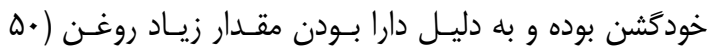

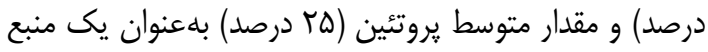

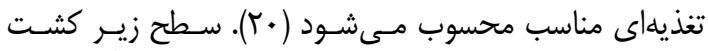

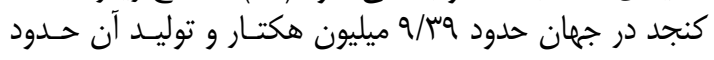

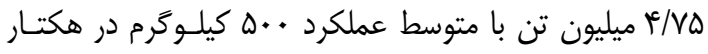

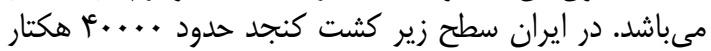

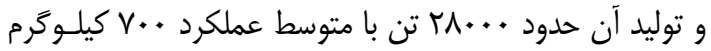

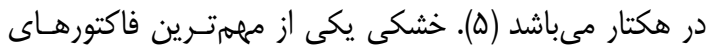

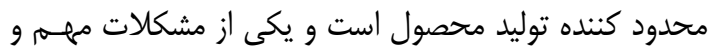

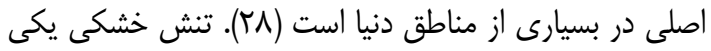

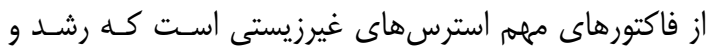

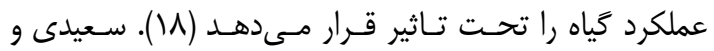

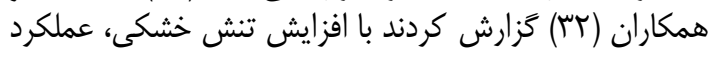

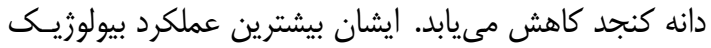

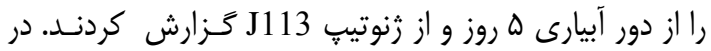

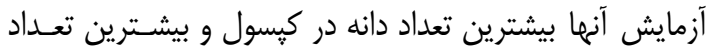

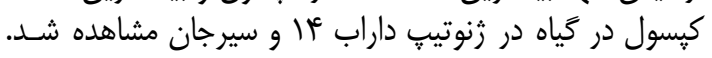

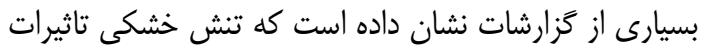

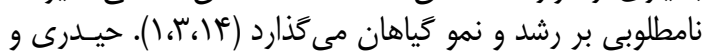

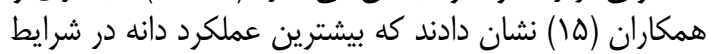

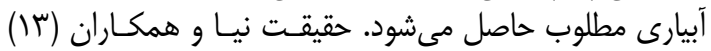
اعلام كردند كه كلنى سازى ميكوريزايى به ويه ويثره توسط كونسه

2- Harmonic Mean

4- Geometric Mean Productivity 5- Yield Index 


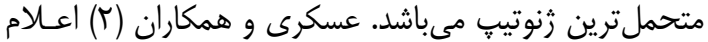

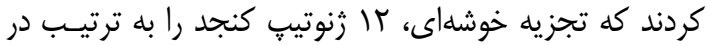

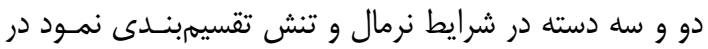

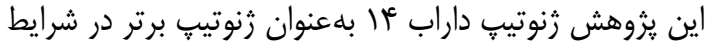

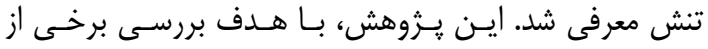

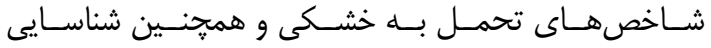

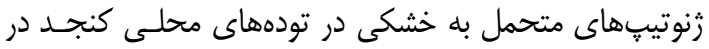

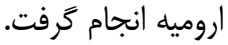

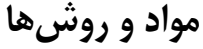

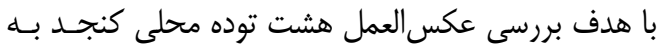

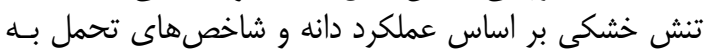

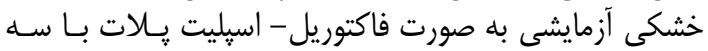

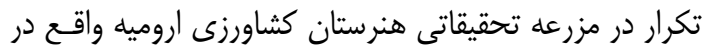

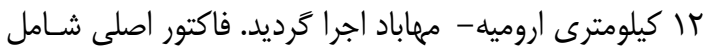

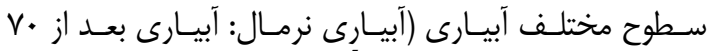

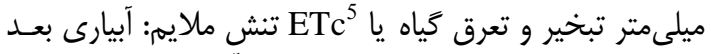

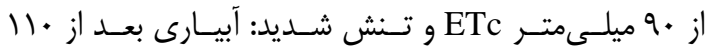

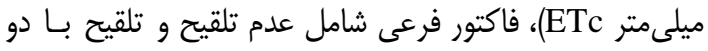

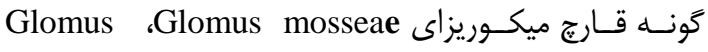
intraradices

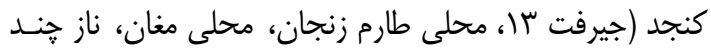

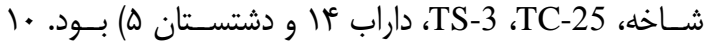

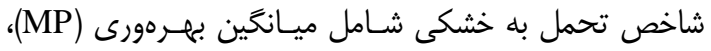

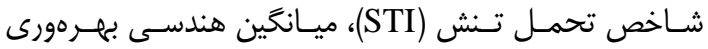

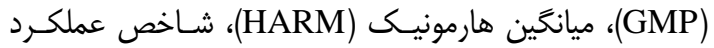

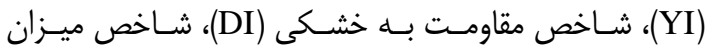

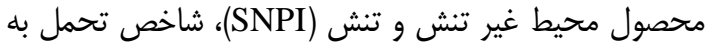

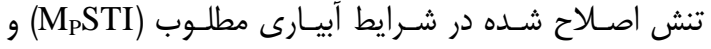

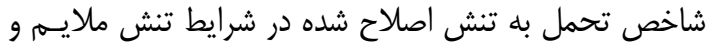
شديد خشكى (M

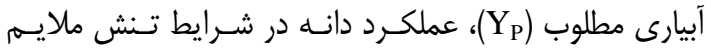

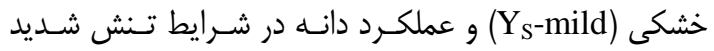

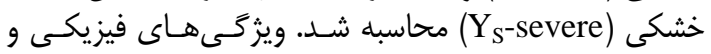

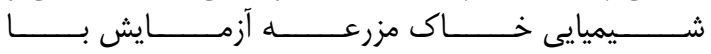

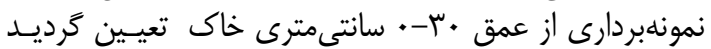

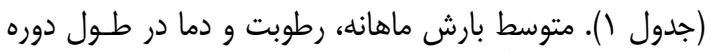

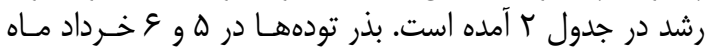

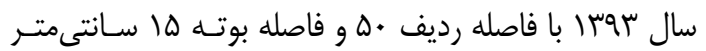

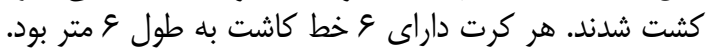

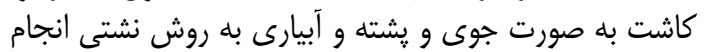

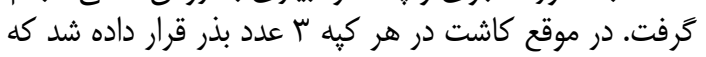

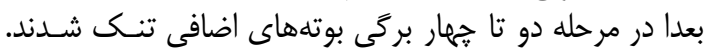

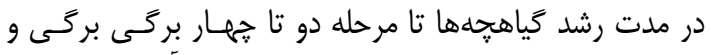

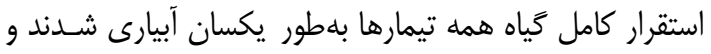

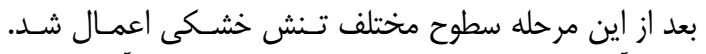

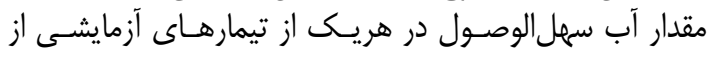
$\mathrm{RAW}=\frac{\mathrm{FC}-\mathrm{PWP}}{100} \times \rho \times \mathrm{D} \times \mathrm{MAD}$
براى رتبهبندى زنوتيِها بر اساس عملكرد در شـرايط تــنش

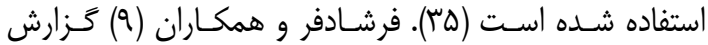

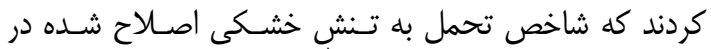

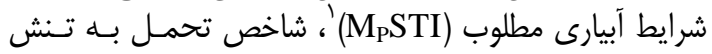

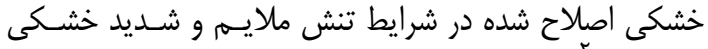

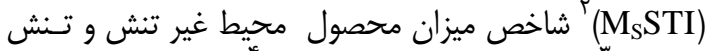

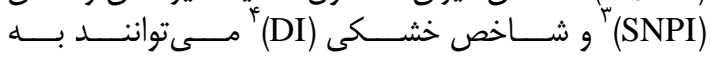

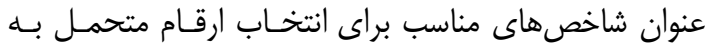

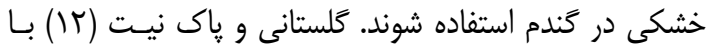

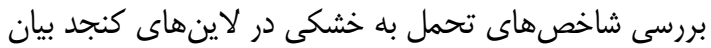

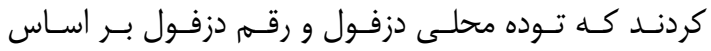

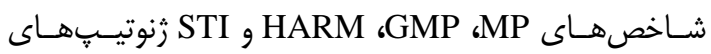

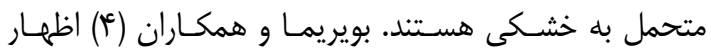

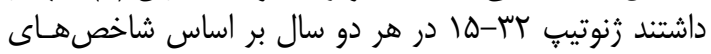

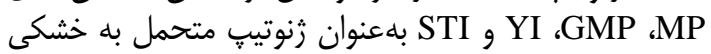

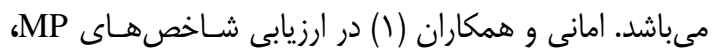

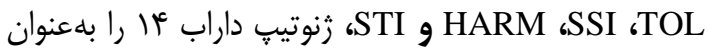

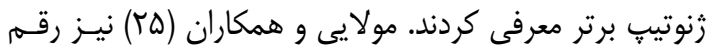

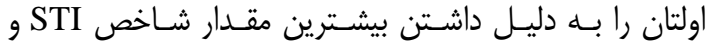

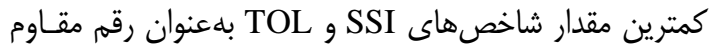

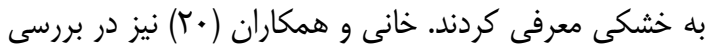

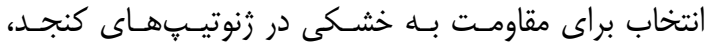

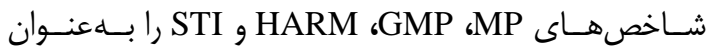

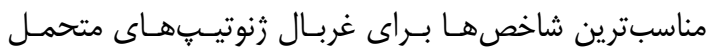

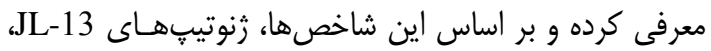

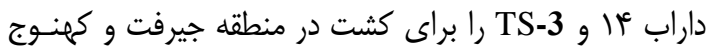

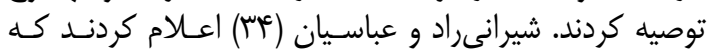

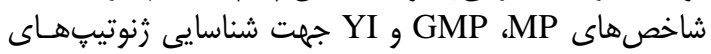

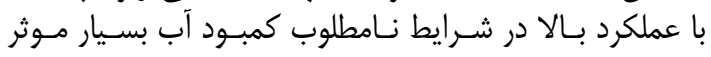

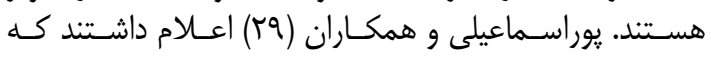

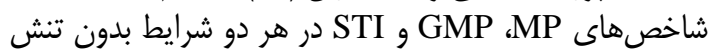

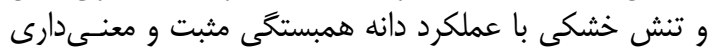

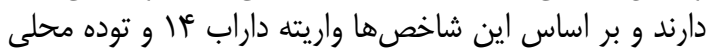

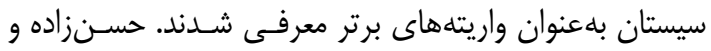

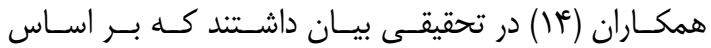

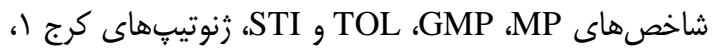

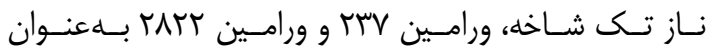

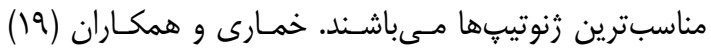

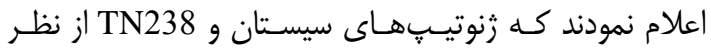

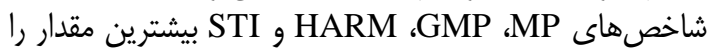

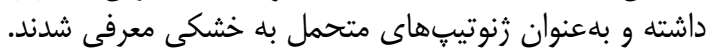

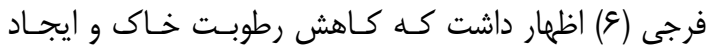

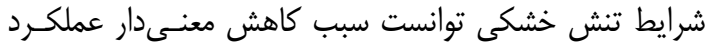

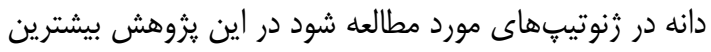

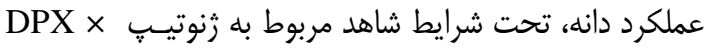

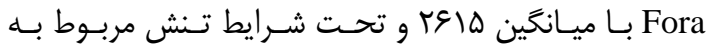

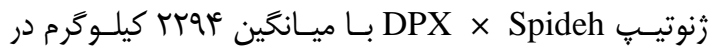

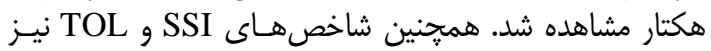

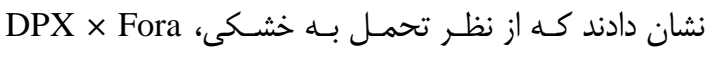

1- Modified Stress Tolerance Index in Optimum Irrigation 2- Modified Stress Tolerance Index in Moderate and Severe Stress 3- Stress Non-Stress Production Index 4- Drought Resistance Index 5- Crop evapotranspiration 


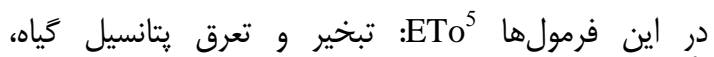
ETp

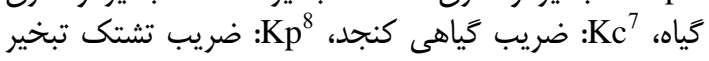

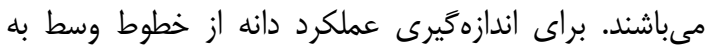

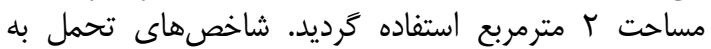

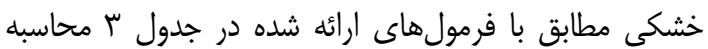
شدند. تجزيه و تحليل واريانس با استفاده از رويه

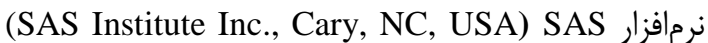

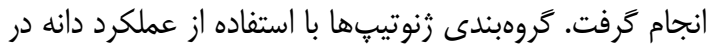

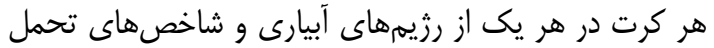

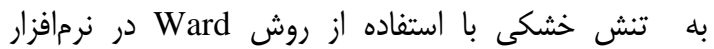
Statgraphics XVI

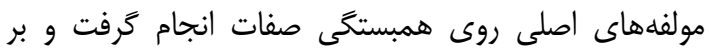
اساس دو مولفه اول تجزيه به مولفههاى اصلى، رونى باى ريلات

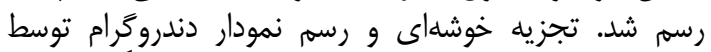

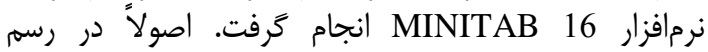

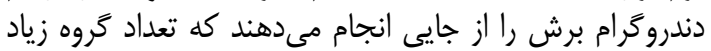

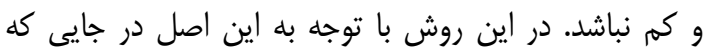
فاصله اتصال تغيير ناخمانى نمود خط برش برش تعيين خرديد.

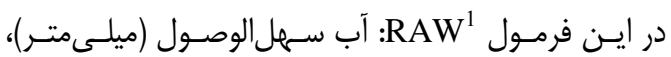
FC

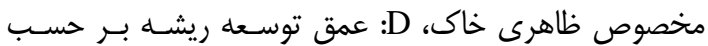

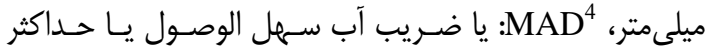

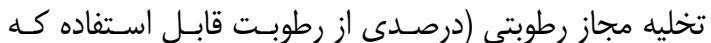

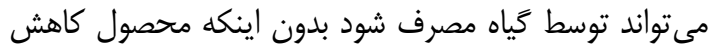

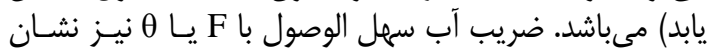

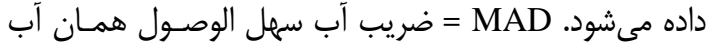

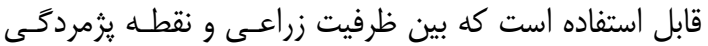

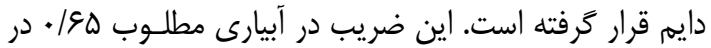

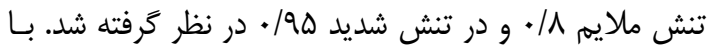

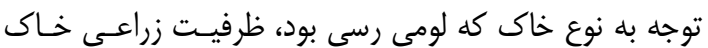

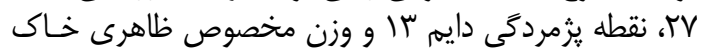

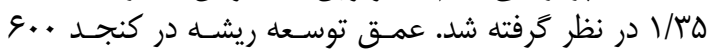

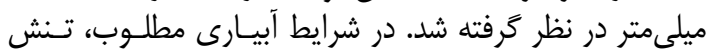

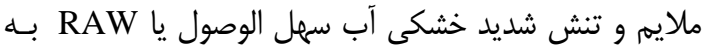

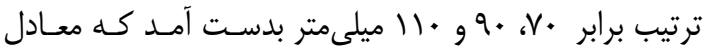

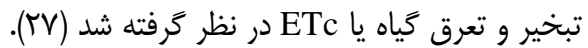

$\mathrm{ETo}=\mathrm{ETp} \times \mathrm{Kp}$

$\mathrm{ETc}=\mathrm{ETo} \times \mathrm{Kc}$

جدول ا - خصوصيات فيزيكى و شيميايى خاك مزرعه آزمايشى در اروميه Table 1. Physical and chemical properties of the soil in the experimental field in Urmia

\begin{tabular}{|c|c|c|c|c|c|c|c|c|c|c|c|c|c|}
\hline $\begin{array}{l}\text { يتاسيم } \\
\text { (ppm) }\end{array}$ & $\begin{array}{l}\text { فسفر } \\
\text { (ppm) }\end{array}$ & $\begin{array}{c}\text { نيتروثن } \\
\text { (\%) }\end{array}$ & كربن & ش) & $\begin{array}{l}\text { سيلت } \\
\text { (\%) }\end{array}$ & $\begin{array}{l}\omega \\
(\%)\end{array}$ & $\begin{array}{l}\text { آهك (\%) } \\
\text { (\%) }\end{array}$ & $\begin{array}{l}\text { دشباع } \\
\text { (\%) }\end{array}$ & درصد مواد خنثى & بي أج & $\begin{array}{c}\text { الكتريكى } \\
\text { EC } \\
(\mathrm{dS} / \mathrm{m}) \\
\end{array}$ & بافت خاى & $\begin{array}{c}\text { عمق خاك } \\
\text { (cm) }\end{array}$ \\
\hline$M T$ & $\Delta / r)$ & $\cdot 111$ & $1 / 11$ & rq & q & rı & - & $r$. & $T r / \Lambda$ & $V / \& q$ & $\cdot / \mathrm{VV}$ & لومى - لومى & $\cdot-r \cdot$ \\
\hline
\end{tabular}

جدول r- دادههاى ماهانه هواشناسى در طى فصل رشد كنجد در اروميه

Table 2. Monthly climate data during sesame growing season in Urmia

\begin{tabular}{|c|c|c|c|c|c|}
\hline \multicolumn{5}{|c|}{ ماه } & \multirow[b]{2}{*}{ جإرامترهاى هواشناسى } \\
\hline شهريور & مرداد & تير & خرداد & ارديبهشت & \\
\hline$r v / \kappa^{c}$ & $\mu \omega / \Gamma$ & $r \Psi / V$ & $T V / \Lambda$ & $T F / I$ & حداكثر دما (درجه سانتى گراد) \\
\hline $\mid r / 9$ & $10 / V$ & $\mid \omega / \Lambda$ & $11 \%$ & $9 / \pi$ & حداقل دما (درجه سانتى كراد) \\
\hline 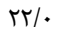 & $r e / F$ & $M F / D$ & $19 / 4$ & $\mid 8 / 8$ & ميانكَين دما (درجه سانتى كراد) \\
\hline$\cdot / 1$ &.$/$ &. & $r \leftarrow / 1$ & $r \Delta / 1$ & مجموع بارندگى (ميلىمتر) \\
\hline 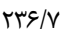 & $r \Lambda / / \Lambda$ & rMN. & $r \Delta \Delta / q$ & $19 \cdot / \pi$ & كل تبخير (ميلى متر) \\
\hline עr & et & $r 4$ & ه) & $\Delta \Gamma$ & ميانكين رطوبت نسبى (٪) \\
\hline
\end{tabular}

\footnotetext{
1- Readily available water 2-Field capacity 3-Permanent wilting point 4- Maximum allowable deficit

5- Reference crop evapotranspiration 6- Pan evapotranspiration $\quad$ 7- Crop coefficient (factor) 8-Pan coefficient (factor)
} 
Table 3. Properties of tolerance indices to drought stress used in current study

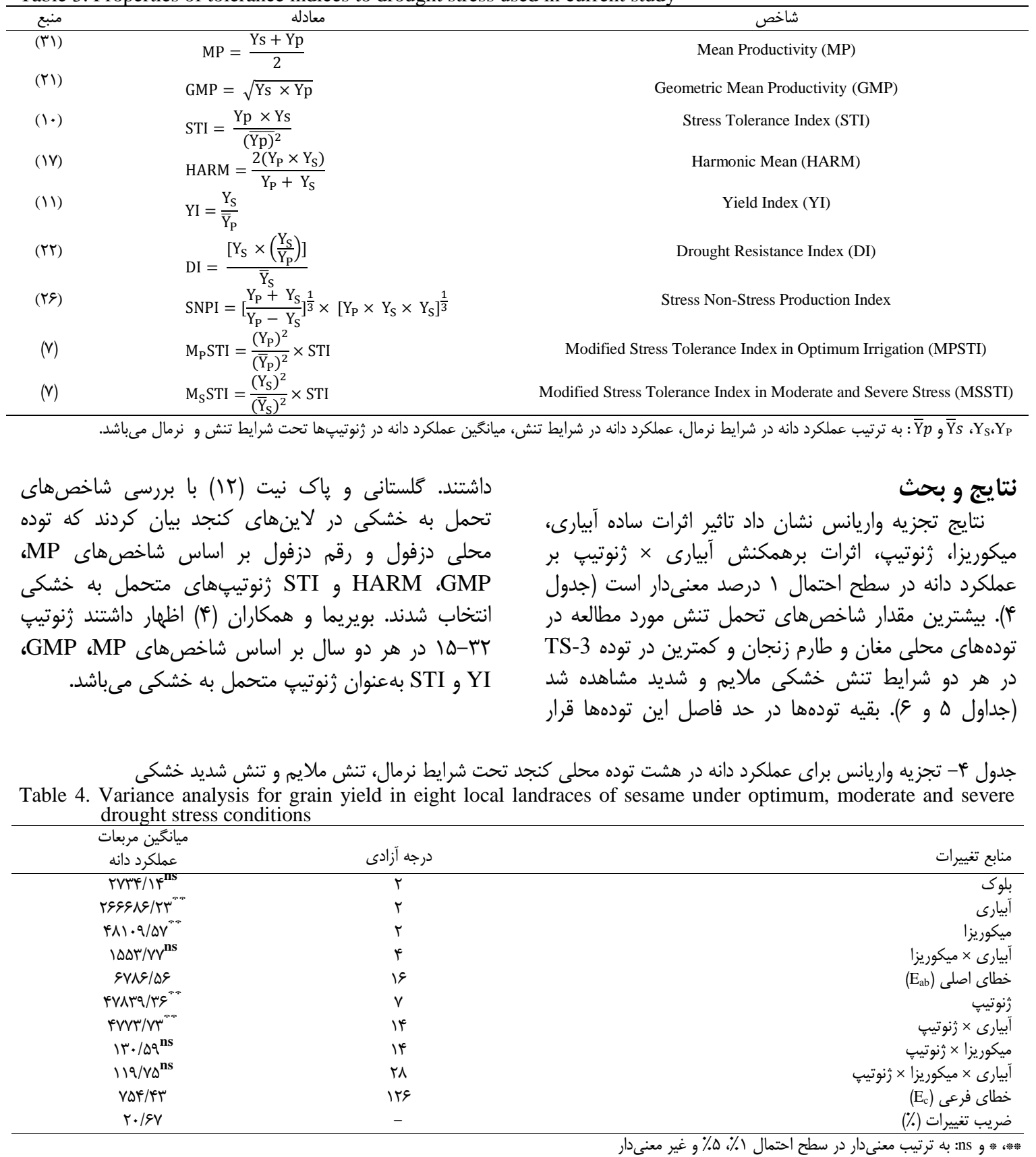


جدول ه- شاخصهاى تحمل تنش در هشت توده محلى كنجد تحت شرايط آبيارى نرمال و تنش ملايم خشكى Table 5. Tolerance indices to drought stress in eight local landraces of sesame under optimum irrigation and moderate

\begin{tabular}{|c|c|c|c|c|c|c|c|c|c|c|c|c|c|}
\hline No & $\begin{array}{c}\text { Local } \\
\text { landraces }\end{array}$ & $\mathrm{Yp}$ & Rank & $\begin{array}{l}\text { Ys- } \\
\text { mild }\end{array}$ & Rank & MP & Rank & STI & Rank & GMP & Rank & HARM & Rank \\
\hline 1 & Jiroft 13 & $1 / \pi$ & V & $1 / \cdot r$ & V & $1 / 11$ & V & g & V & $1 / I V$ & V & $1 / 19$ & $\mathrm{v}$ \\
\hline r & $\begin{array}{l}\text { Zanjan Tarom } \\
\text { landrace }\end{array}$ & $r / 9$. & r & $1 / v e$ & r & $T / M$ & r & $1 / \pi r$ & r & $r / N F$ & r & $r / l$. & r \\
\hline r & $\begin{array}{l}\text { Moghan } \\
\text { landrace }\end{array}$ & $\Gamma / \Lambda \Lambda$ & 1 & $1 / M$ & 1 & $r / \Delta r$ & 1 & $1 / 09$ & 1 & $r / F T^{2}$ & 1 & ( & 1 \\
\hline r & $\begin{array}{l}\text { Naz of several } \\
\text { branches }\end{array}$ & $1 / 4 V$ & 8 & $1 / M$ & 8 & $1 / \mu$. & 8 &.$/ 4 F$ & 8 & $1 / 79$ & 8 & $1 / T \Lambda$ & 8 \\
\hline$\Delta$ & TC-25 & $r / M \Lambda$ & r & $1 / 41$ & r & $1 / v 9$ & r & . $/ A r$ & r & $1 / v \Delta$ & r & $1 / N 1$ & $r$ \\
\hline 9 & TS-3 & $1 / \pi$ & $\wedge$ & .198 & $\wedge$ & $1 / \cdot 9$ & $\wedge$ & . & $\wedge$ & $1 / \cdot 1$ & $\wedge$ & $1 / * V$ & $\Lambda$ \\
\hline$v$ & Darab 14 & I/AT & r & $1 / r \Lambda$ & r & $1 / \Delta \Delta$ & f &. $\mid q T$ & f & V & f & $1 / \Delta$. & f \\
\hline$\wedge$ & Dashtestan 5 & $1 / 9 \Lambda$ & $\Delta$ & $1 / T^{c}$ & $\Delta$ & $1 / 4$ & $\Delta$ & .109 & $\Delta$ & $1 / 4 \varphi^{4}$ & $\Delta$ & T/א & $\Delta$ \\
\hline
\end{tabular}

Table 5. Continued

\begin{tabular}{|c|c|c|c|c|c|c|c|c|c|c|c|}
\hline No & Local landraces & SNPI & Rank & YI & Rank & DI & Rank & $\mathrm{M}_{\mathrm{P}} \mathrm{STI}$ & Rank & $\mathrm{M}_{S} \mathrm{STI}$ & Rank \\
\hline 1 & Jiroft 13 & $T / 4)$ & $\mathrm{V}$ & $\cdot / \Delta \mu$ & $\mathrm{V}$ & $\cdot / 9$. & $\mathrm{V}$ &.$/ \mathrm{IV}$ & $\mathrm{V}$ &.$/ 4 t$ & $\mathrm{~V}$ \\
\hline$r$ & Zanjan Tarom landrace & $m / 4$ & r &.$/ 91$ & r & $\cdot / 19$ & 1 & $r / 4$ & $r$ & $r / I f$ & r \\
\hline r & Naz of several branches & $r / \mathcal{L}$. & $\varepsilon$ & $\cdot / \Delta \Lambda$ & $\varepsilon$ & $\cdot / 90$ & $\varepsilon$ & . & $\varepsilon$ & 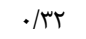 & 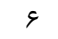 \\
\hline$\Delta$ & TC-25 & $r / 9 q$ & r & $\cdot / V^{m}$ & r & $\cdot \mid \& \Lambda$ & $r / \Delta$ & $1 / \cdot f^{f}$ & r & ./94 & 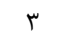 \\
\hline 9 & TS-3 & $r / \cdot \Lambda$ & $\wedge$ & $\cdot / 0$ & $\wedge$ & $\cdot 1 \Delta 9$ & $\wedge$ &.$/ 14$ & $\wedge$ &.$/ 19$ & $\wedge$ \\
\hline$\wedge$ & Dashtestan 5 & $r / \Delta \Delta$ & $\Delta$ & $\cdot|g|^{2}$ & $\Delta$ &.$/ 99$ & r & . Rt & $\Delta$ &.$/ 19 q$ & $\Delta$ \\
\hline
\end{tabular}

جدول و- شاخصهاى تحمل تنش در هشت توده محلى كنجد تحت شرايط آيبارى نرمال و تنش شديد خشكى

Table 6. Tolerance indices to drought stress in eight local landraces of sesame under optimum irrigation and severe

\begin{tabular}{|c|c|c|c|c|c|c|c|c|c|c|c|c|c|}
\hline No & $\begin{array}{c}\text { Local } \\
\text { landraces }\end{array}$ & $\mathrm{Yp}$ & Rank & $\begin{array}{l}\text { Ys- } \\
\text { severe }\end{array}$ & Rank & MP & Rank & STI & Rank & GMP & Rank & HARM & Rank \\
\hline 1 & Jiroft 13 & سז/I & V &.$/$ sq $^{\mathrm{a}}$ & $V$ & $\cdot / \mathrm{MA}$ & $V$ &.$/ 19$ & $V$ & $\cdot / V \&$ & $V$ & .199 & $V$ \\
\hline$r$ & $\begin{array}{c}\text { Zanjan Tarom } \\
\text { landrace }\end{array}$ & $r / \varepsilon$. & $r$ & .191 & r & $1 / v 9$ & r & .191 & r & $1 / 9$. & r & $1 / 4$ & r \\
\hline r & $\begin{array}{l}\text { Moghan } \\
\text { landrace }\end{array}$ & $\mathrm{m} / \Lambda$ & 1 & سו & 1 & $r / 19$ & 1 & .199 & 1 & $1 / 9$. & 1 & $1 / 9 V$ & 1 \\
\hline r & $\begin{array}{c}\text { Naz of } \\
\text { several } \\
\text { branches }\end{array}$ & $1 / 4 V$ & 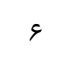 & $\cdot \mid \Delta s$ & $\varepsilon$ & $1 / \cdot 1$ & $\varepsilon$ & T4/ & 8 &.$/ 91$ & $\varepsilon$ & $\cdot|\wedge|$ & 9 \\
\hline$\Delta$ & TC-25 & $r / M$ & r & $\cdot / \Lambda F$ & r & $1 / \Delta 1$ & r &.$/ 1 q q$ & r & $1 / \% \Delta$ & r & $\mid / r 1$ & r \\
\hline 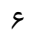 & TS-3 & I/Tr & $\wedge$ & $\cdot|c|$ & $\wedge$ & $\cdot|\Lambda|$ & $\wedge$ & سו/. & $\wedge$ & $\cdot / \mathrm{V}$ & $\wedge$ &.$|9|$ & $\wedge$ \\
\hline$v$ & Darab 14 & V/AT & r & $\cdot / V \Delta$ & r & $1 / 4 \Lambda$ & r & عس/. & r & I/IV & f & $1 / .9$ & f \\
\hline$\Lambda$ & Dashtestan 5 & $1 / 9 \wedge$ & $\Delta$ & س & $\Delta$ & $1 / 19$ & $\Delta$ & $\cdot / T \Lambda$ & $\Delta$ & 1.r & $\Delta$ &.$/ 94$ & $\Delta$ \\
\hline
\end{tabular}

Table 6. Continued

\begin{tabular}{|c|c|c|c|c|c|c|c|c|c|c|c|}
\hline No & Local landraces & SNPI & Rank & YI & Rank & DI & Rank & MPSTI & Rank & $\mathrm{M}_{\mathrm{S}} \mathrm{STI}$ & Rank \\
\hline 1 & Jiroft 13 & $\cdot / \Lambda$ & $\mathrm{V}$ & T/R & V & $\cdot / 4$ & $\mathrm{~V}$ & $\cdot 1 \cdot V$ & $\mathrm{~V}$ & .1 .9 & V \\
\hline r & Zanjan Tarom landrace & $1 / \mathrm{VV}$ & r & $\cdot|\Delta|$ & t & $\cdot|\Delta|$ & r & $1 / T F$ & r & $1 / 4 q$ & r \\
\hline r & Moghan landrace & $r / \cdot \Delta$ & 1 & .109 & 1 & $\cdot \mid \Delta \varphi$ & 1 & $r / 8 \mid$ & 1 & T & 1 \\
\hline r & Naz of several branches & $1 / \cdot 1$ & 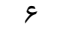 &.$/ 4 q$ & 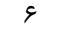 & $\cdot / \mu \cdot$ & द & - (1) & द & $\cdot / 1 f$ & 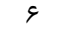 \\
\hline$\Delta$ & TC-25 & $1 / \Delta 1$ & r & سז/. & r & $\cdot / t^{2}$ & r & . & r & $\cdot / 9 V$ & r \\
\hline$\varepsilon$ & TS-3 & $\cdot / V^{c}$ & $\wedge$ & $\cdot / 4 \mid$ & $\wedge$ & .119 & $\wedge$ & $.1 \cdot \Delta$ & $\wedge$ & $\cdot 1 \cdot \mathrm{f}^{\mathrm{f}}$ & $\wedge$ \\
\hline
\end{tabular}

سيستان به عنوان واريتههاى برتر معرفى شدند. در نمودار باى

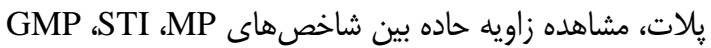

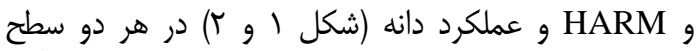

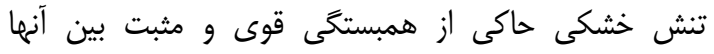

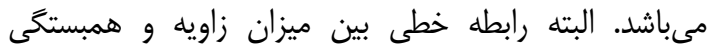

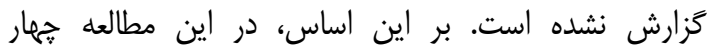

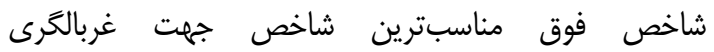

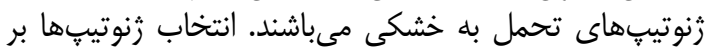

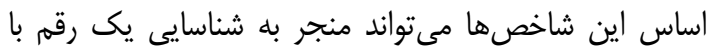

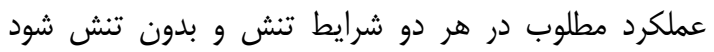

.(A

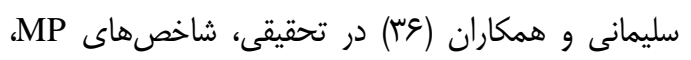

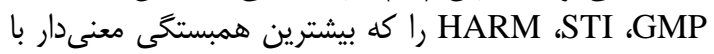
عملكرد دانه در شرايط بدون تنش و تنش داشتند به به عنوان

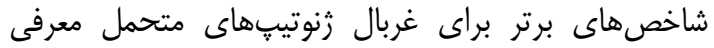

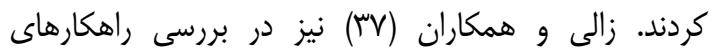

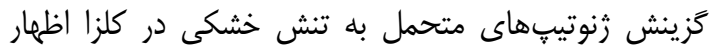

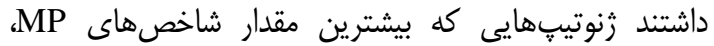

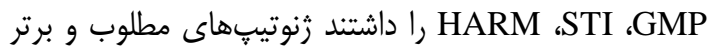
شناخته شدند. يوراسماعيلى و همكاران (

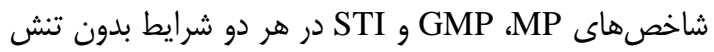

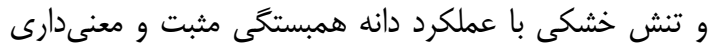

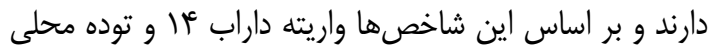


19. بررسى تحمل به خشكى در تودههاى محلى كنجد

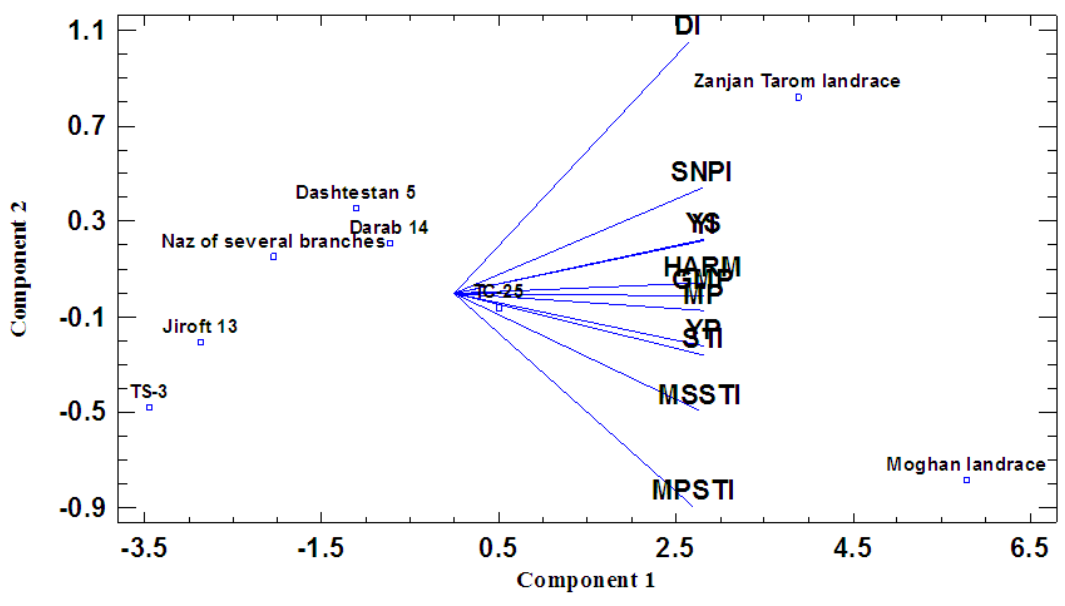

شكل ا - باى پالات براى تعيين زنوتيِها و شاخصهاى برتر در هشت توده محلى كنجد در شرايط تنش ملايم خشكى

Figure 1. Biplot for determing genotypes and superior indices in eight local landraces of sesame in moderate drought stress condition

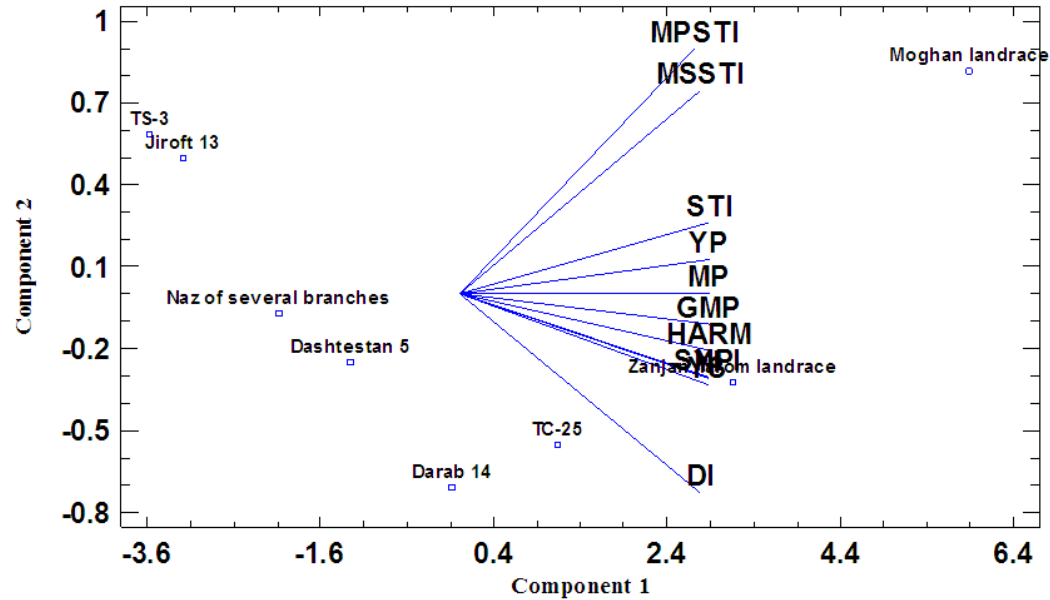

شكل r- باى يلات براى تعيين زنوتيٍها و شاخصهاى برتر در هشت توده محلى كنجد در شرايط تنش شديد خشكى

Figure 2. Biplot for determing genotypes and superior indices in eight local landraces of sesame in severe drought stress condition

ملايم و شديد، عملكرد دانه بالايى داشتند بهاعنوان رقم

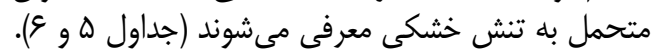
تجزيه خوشهاى به تنش

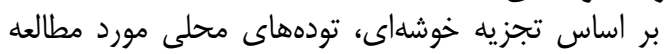

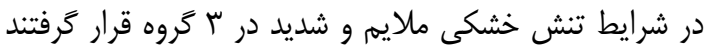

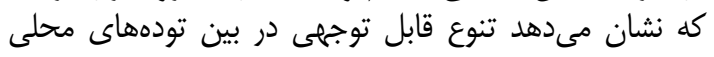

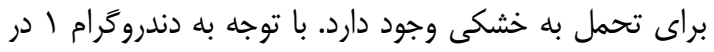

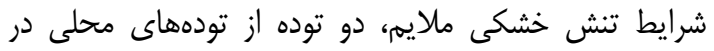

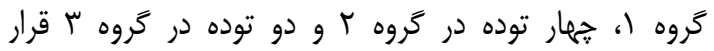

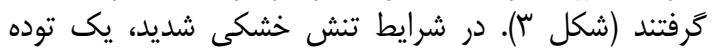

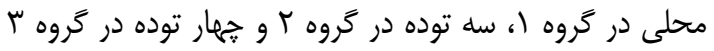

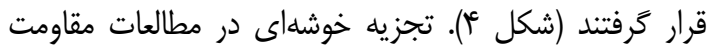

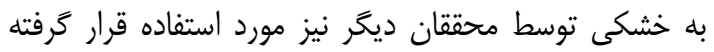

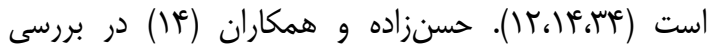

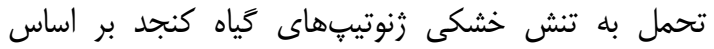

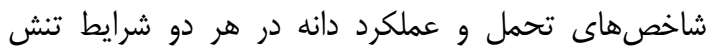

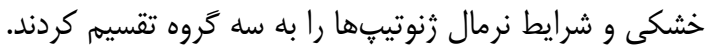

در شرايط تنش خشكى ملايه، تودههاى محلى مغان بان بار

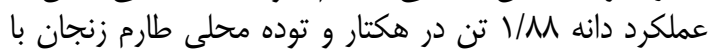

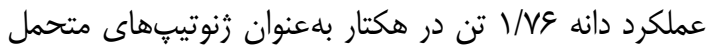

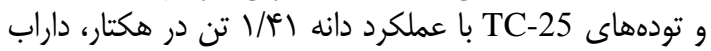

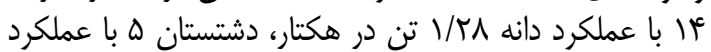

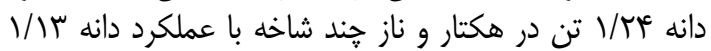

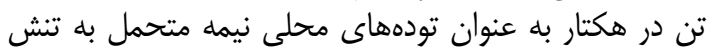

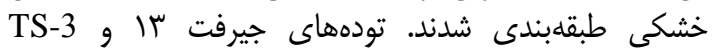

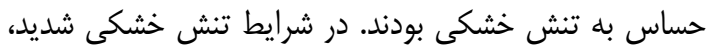

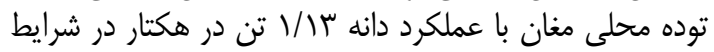

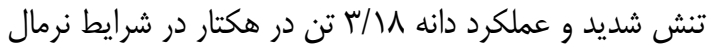

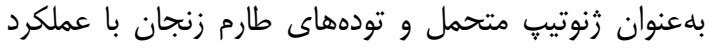

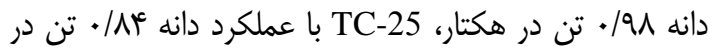

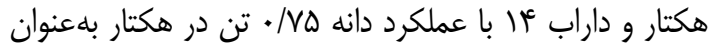

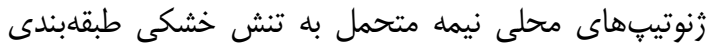

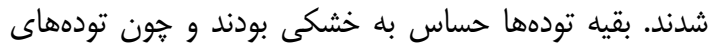

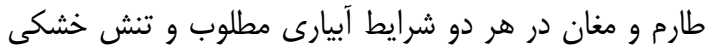




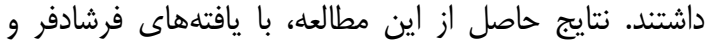

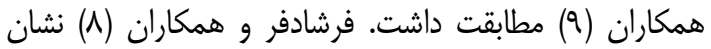

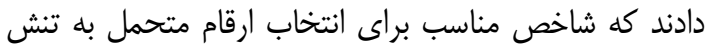

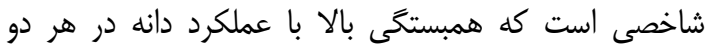

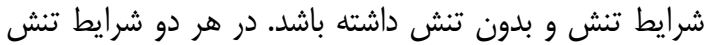

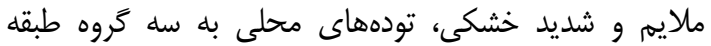

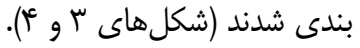

كلستانى و ياك نيت (T) (I) نشان دادند كه بر اساس تجزيه

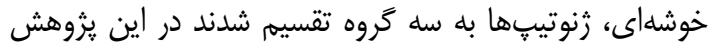

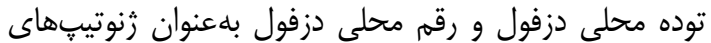

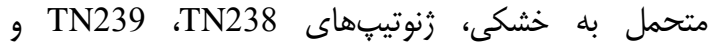

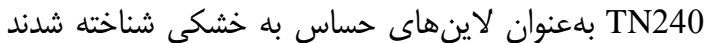

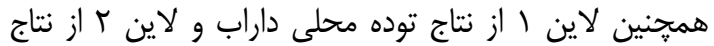

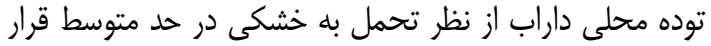

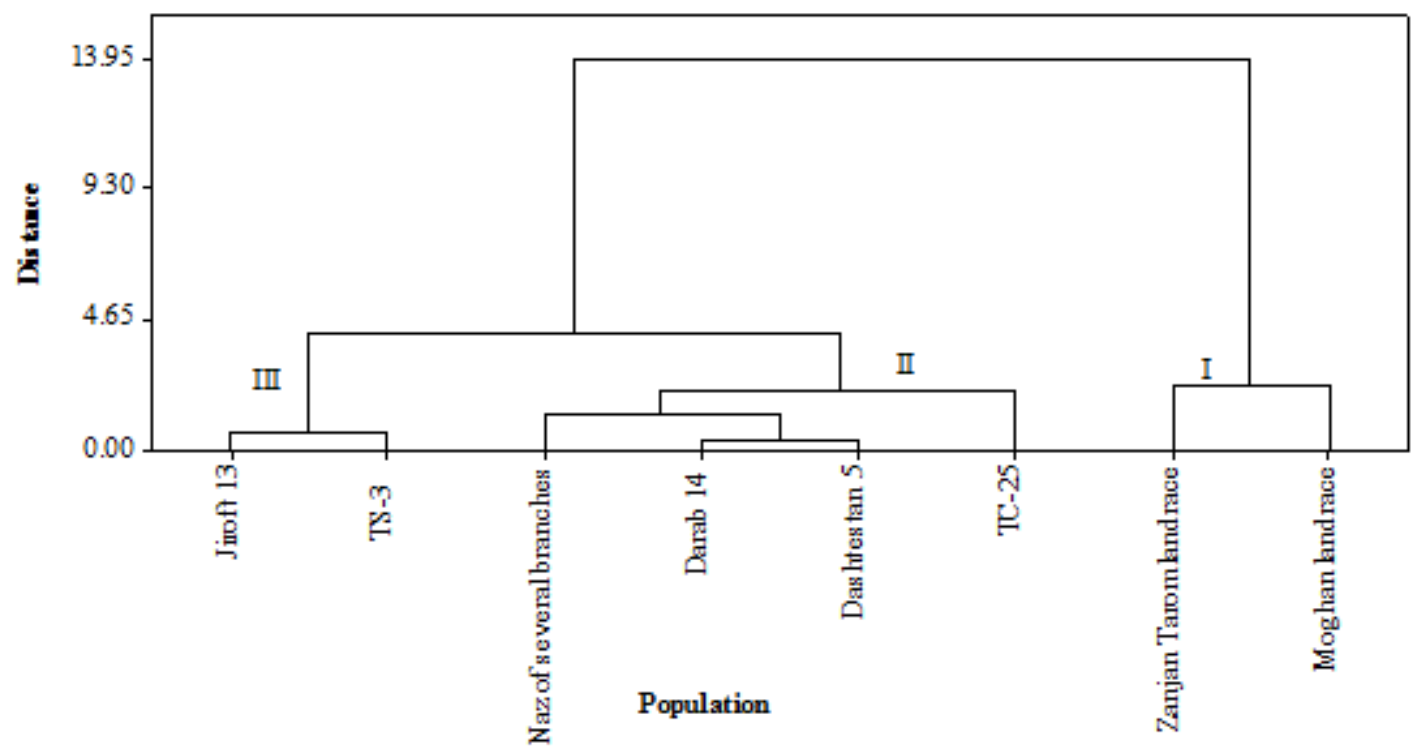

شكل س- دندروكرام تجزيه خوشهاى هشت توده محلى كنجد بر اساس روش Ward روى شاخصهاى تحمل به خشكى در شرايط تنش ملايم

Figure 3. Dendrogram of cluster analysis of eight local landraces of sesame basis of Ward method on tolerance indices to drought stress in moderate drought stress

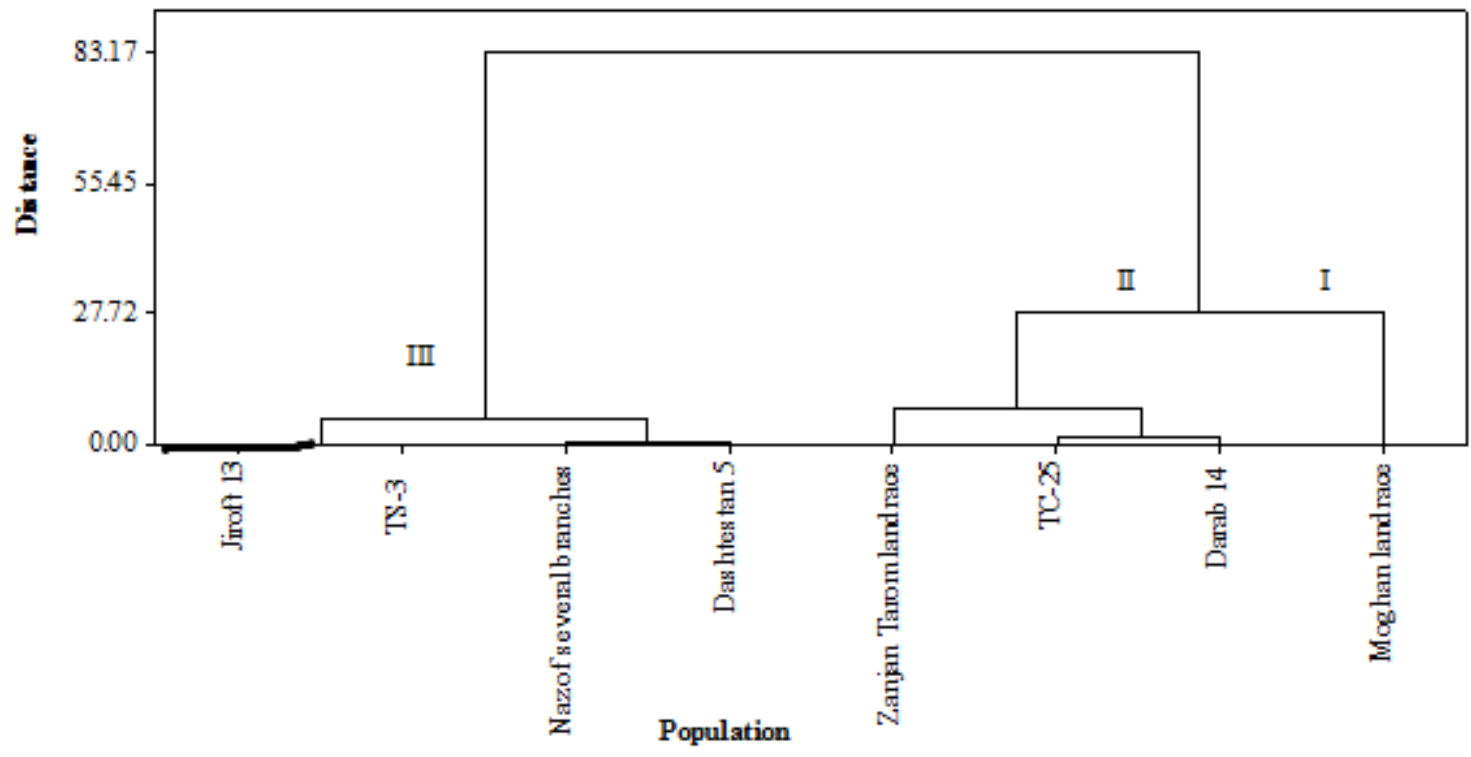

شكل ع - دندروگرام تجزيه خوشهاى هشت توده محلى كنجد بر اساس روش Ward روى شاخصهاى تحمل به خشكى در شرايط تنش شديد Figure 4. Dendrogram of cluster analysis of eight local landraces of sesame sesame basis of Ward method on tolerance indices to drought stress in severe drought stress 


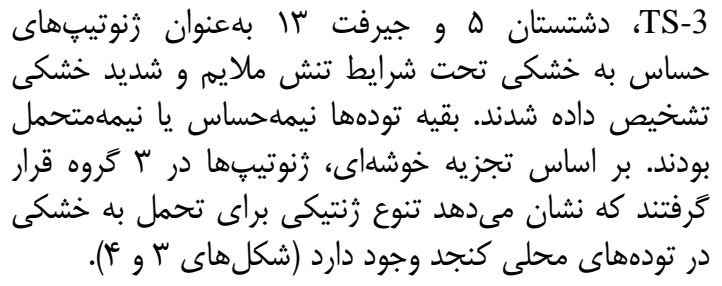

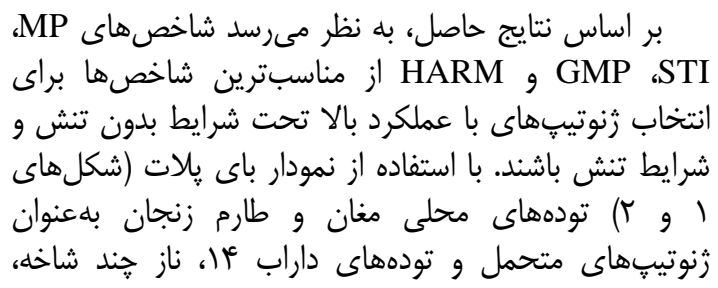

منابع

1. Amani, M., P. Golkar and G. Mohammadi-Nejad. 2012. Evaluation of drought tolerance in different genotypes of sesame (Sesame indicum L.). International Journal of Recent Scientific Research, 3: 226230.

2. Askari, A., M. Zabet, M.G. Ghaderi and A. Shorvazdi. 2016. Choose the most important traits affecting on yield of some sesame genotypes (Sesamum indicum L.) in normal and stress conditions. Journal of Crop Breeding, 8: 78-87 (In Persian).

3. Bahrami, H., J. Razmjoo and A. Ostadi Jafari. 2012. Effect of drought stress on germination and seedling growth of sesame cultivars (Sesamum indicum L.). International Journal of Agriculture Science, 2: 423-428.

4. Boureima, S., M. Diouf, A.I. Amoukou and P. Van Damme. 2016. Screening for ources of tolerance to drought in sesame induced mutants: Assessment of indirect selection criteria for seed yield. International Journal of Pure and Applied Biosience, 4: 45-60.

5. Food and Agricultural Organization. 2013. FAO 2013 [WWW.document]. http://faostat3.fao.org.

6. Faraji, F. 2016. Evaluation of some soybean genotypes (Glycine max) under salt stress. Journal of Crop Breeding, 8: 30-36 (In Persian).

7. Farshadfar, E. and J. Sutka. 2002. Screening drought tolerance criteria in maize. Acta Agronomica Hungarica, 50: 411-416.

8. Farshadfar, E., M. Ghannadha, M. Zahravi and J. Sutka. 2001. Genetic analysis of drought tolerance in wheat. Plant Breeding, 114: 542-544.

9. Farshadfar, E., M.M. Poursiahbidi and S.M. Safavi. 2013. Assessment of drought tolerance in land races of bread wheat based on resistance/tolerance indices. International Journal of Advanced Biological and Biomedical Research, 1: 143-158.

10. Fernandez, G.C.J. 1992. Effective selection criteria for assessing stress tolerance. In: Kuo C.G. (Ed.), Proceedings of the International Symposium on Adaptation of Vegetables and Other Food Crops in Temperature and Water Stress, pp: 257-270.

11. Gavuzzi, P., F. Rizza, M. Palumbo, R.G. Campaline, G.L. Ricciardi and B. Borghi. 1997. Evaluation of field and laboratory predictors of drought and heat tolerance in winter cereals. Canadian Journal of Plant Science, 77: 523-531.

12. Golestani, M. and H. Pakniat. 2007. Evaluation of drought tolerance indices in sesame lines. Journal of Scientific and Technological Agriculture, 11: 141-149 (In Persian).

13. Haghighatnia, H., H. Nadian, F. Rejali and A.R. Tavakoli. 2012. Effect of two species of arbuscularmycorrhizal fungi on vegetative growth and phosphorous uptake of Mexican lime rootstock (Citrus aurantifolia) under drought stress conditions. Seed and Plant Improvement Journal, 2: 403-417 (In Persian).

14. Hassanzadeh, M., A. Ashari, S.H. Jamaati-e-Somarin, M. Saeidi, R. Zabihi-e-Mahmoodabad and S. Hokmalipour. 2009. Effects of water deficit on drought tolerance indices of sesame (Sesamum indicum L.) genotypes in Moghan Region. Research Journal of Environmental Sciences, 3: 116-121.

15. Heidari, M., M. Galavi and M. Hassani. 2011. Effect of sulfur and iron fertilizers on yield, yield components and nutrient uptake in sesame (Sesamum indicum L.) under water stress. African Journal of Biotechnology, 10: 816-8822.

16. Izanloo, A., H. Zeinali, A. Hosainzadeh and N. Majnoon Hoseini. 2002. Determination the best indicators of drought tolerance in soybean cultivars. The 7th congress Agronomy Sciences and Plant Breeding. Karaj. Iran, 553-554 (In Persian).

17. Jafari, A., F. Paknejad and M. Al-Ahmadi. 2009. Evaluation of selection indices for drought tolerance of corn (Zea mays L.) hybrids. International Journal of Plant Production, 3: 33-38.

18. Jaleel, C.A., P. Manivannan, A. Wahid, M. Farooq, H.J. Al-Juburi, R. Somasundaram and R. Panneersel Vam. 2009. Drought Stress in Plants: A Review on Morphological Characteristics and Pigments Composition. Inernational Journal of Agriculture and Biology, 11: 100-105.

19. Khammari, M., A. Ghanbari and H. Rostami. 2013. Evaluation indicator of drought stress in different cultivars of sesame. International Journal of Management Sciences and Business Research, 2: 22268235.

20. Khani, M.R., H. Heidari Sharifabad, H. Madani, G.H. Noor Mohamadi and F. Darvish. 2010. Selection for tolerance to drought in sesame genotype. The new findings Agriculture, 4: 347-359 (In Persian).

21. Kristin, A.S., R.R. Serna, F.I. Perez, B.C. Enriquez, J.A.A. Gallegos, P.R. Vallejo, N. Wassimi and J.D. Kelly. 1997. Improving common bean performance under drought stress. Crop Science, 37: 5160.

22. Lan, J. 1998. Comparison of evaluating methods for agronomic drought resistance in crops. Acta Agriculturae Boreali-occidentalis Sinica, 7: 85-87. 
23. Ledent, J.F. and O.N. Moss. 1979. Relation of morphological characters and shoot yield in wheat. Crop Science, 19: 445-451.

24. Mensah, J.K., B.O. Obadoni, P. Eruotor and F. Onome-Trieguna. 2006. Simulated flooding and drought effects on germination, growth and yield parameters of sesame. African Journal Biotechnology, 13: 1249-1253.

25. Molaei, P., A. Ebadi, A. Namvar and T. Khandan Bejandi. 2012. Water relation, solute accumulation and cell membrane injury in sesame (Sesamum indicum L.) cultivars subjected to water stress. Annals of Biological Research, 3: 1833-1838.

26. Moosavi, S.S., B. Yazdi Samadi, M.R. Naghavi, A.A. Zali, H. Dashtid and A. Pourshahbazi. 2008. Introduction of new indices to identify relative drought tolerance and resistance in wheat genotypes. Desert, 12: 165-178 (In Persian).

27. Mousavi, S.F. and S. Akhavan. 2007. Irrigation Principles, Press Kankash, 415 (In Persian).

28. Passioura, J.B. 2007. The drought environment: physical, biological and agricultural perspectives. Journal of Experimental Botany, 58: 113-117.

29. Poor-Esmaeili, H.A., H.R. Fanaeib and M.H. Saberic. 2014. Evaluation of drought tolerant cultivars and lines of sesame using stress tolerance indices. Scientific Journal of Crop Science, 3: 66-70.

30. Richards, R.A. 1996. Defining selection criteria to improve yield under drought. Plant Growth Regulators, 20: 157-166.

31. Rosielle, A.A. and J. Hamblin. 1981. Theoretical aspects of selection for yield in stress and non- stress environment. Crop Science, 21: 943-946.

32. Saeidi, A., E. Tohidi-Nezhad, F. Ebrahimi, G. Mohammadi-Nejad and M.H. Shirzadi. 2012. Investigation of water stress on yield and some yield components of sesame genotypes in Jiroft region. Journal of Applied Sciences Research, 8: 243-246.

33. Schnider, K.A., R. Rosales-Serna, F. Ibarra-Perez, B. Cazares-Enriques, J.A. Acosta-Gallegos, P. Ramirez-Vallejo, N. Wassimi and J.D. Kelly. 1997. Improving common bean performance under drought stress. Crop Science, 37: 43-50.

34. Shiranirad, A.H. and A. Abbasian. 2011. Evaluation of Drought Tolerance in Rapeseed Genotypes under Non Stress and Drought Stress Conditions. Notulae Botanici Horti Agrobotanici Cluj-Napoca, 39: 164-171.

35. Sio-Se Mardeh, A., A. Ahmadi, K. Poustini and V. Mohammadi. 2006. Evaluation of drought resistance indices under various environmental conditions. Field Crop Reseasrch, 98: 222-229.

36. Soleimani, A., M.R. Bihamta, S.A. Peyghambari and R. Maali-Amiri. 2017. Evaluation of late season drought in barley genotypes using some drought tolerance indices. Journal of Crop Breeding, 9(23): 166-176.

37. Zali, H., T. Hasanloo, O. Sofalian, A. Asghari and M. Zeinalabedini. 2016. Appropriate strategies for selection of drought tolerant genotypes in Canola. Journal of Crop Breeding, 8(20): 77-90. 


\title{
Investigation the Drought Tollerance of Sesame (Sesamium indicum L.) Local Landraces Based on Drought Stress Tolerance Indices in Different Levels of Irrigation and Mycorrhizae
}

\author{
Esmaeil Gholinezhad ${ }^{1}$ and Reza Darvishzadeh ${ }^{2}$
}

\author{
1- Associate Professor, Department of Agricultural Sciences, Payame Noor University, Tehran, Iran \\ (Corresponding Author: gholinezhad1358@yahoo.com) \\ 2- Professor, Department of Plant Breeding and Biotechnology, Urmia University, Urmia, Iran \\ Received: February 2, 2017 \\ Accepted: June 3, 2017
}

\begin{abstract}
Sesame is one of the plants that due to the high content (47-52\%) and high quality (low cholesterol and some antioxidants) its seed oil, important role has in human health. An experimental with objective to evaluate eight local landraces of sesame to drought stress based on grain yield and drought tolerance indices using factorial split plot design was conducted with three replications in research field of Urmia agricultural high school. The main factor was consisted different levels of irrigation, normal irrigation (irrigation after $70 \mathrm{~mm}$ evaporation of crop (ETc)), moderate drought stress (irrigation after $90 \mathrm{~mm} \mathrm{ETc)}$ and severe drought stress (irrigation after $110 \mathrm{~mm} \mathrm{ETc}$ ), sub plots including two kinds of mycorrhizae fungi Glomus mosseae, Glomus intraradices and non-inoculated (control). Sub-sub plots consisted of eight local landraces of sesame with names Jiroft13, Zanjan Tarom landrace, Moghan landrace, Naz of several branches, TC-25, TS-3, Darab 14 and Dashtestan 5. Ten drought tolerance indices including mean productivity (MP), geometric mean productivity (GMP), stress tolerance index (STI), harmonic mean (HARM), yield index (YI), drought resistance index (DI), stress nonstress production index (SNPI), modified stress tolerance index in optimum irrigation (MPSTI) and modified stress tolerance index in moderate and severe stress (MSSTI) were calculated based on grain yield under well-watered (YP), moderate drought stress (YS-mild) and severe drought stress (YS-severe) conditions. Based on cluster analysis, the studied local landraces were grouped in 3 clusters in each one of water treatment conditions. Based on biplot analysis, local landraces Moghan landrace and Zanjan Tarom landrace as resistant genotypes and landrace TC-25 were classified as moderately resistant and other local landraces were somewhat susceptible.
\end{abstract}

Keywords: Biplot, Cluster analysis, Drought stress, Local landraces, Sesame 